\title{
The Role of Sperm Chromatin Integrity and DNA Damage on Male Infertility
}

\author{
Aspinder Singh and Ashok Agarwal* \\ Center for Reproductive Medicine, Cleveland Clinic, Cleveland, Ohio, USA
}

\begin{abstract}
Infertility affects approximately a quarter of all couples. Of these cases, roughly half are due to male factors. However, more than $50 \%$ of the causes of male factor infertility are still obscure. Contemporary Andrology includes a thorough analysis of the sperm looking at the cellular and subcellular imperfections which may have an adverse effect on fertility. Defects in DNA and chromatin structure are examples of such analysis. The structure of spermatozoa DNA is very unique, highly specialized in order to control time-appropriate maturation of the zygote. Damage to sperm DNA may occur as a result of intrinsic factors such as limited defenses against oxidative stress, ageing and varicocele, or as a result of extrinsic determinants such as medications and environmental factors. This damage thereby may have negative effects on ART procedures, and could lead to failure of fertilization. Sperm DNA damage significantly contributes to the growing number of infertility cases, and should be a part of a modern andrology lab.
\end{abstract}

Keywords: Sperm, DNA, chromatin, protamine, DNA repair, male infertility.

\section{INTRODUCTION}

Infertility or involuntary childlessness may not be often thought as a leading disease when considering ailments affecting the general human populations. However, infertility is a common disease, affecting between 17 and $25 \%$ of all couples and is defined as the inability of a couple in reproductive age to conceive following 12 months of unprotected intercourse. Infertility may have a lasting impact on the health and relationship of the affected individuals [1]. When approaching such problem, traditionally the female partner is scrutinized. However, of all infertility cases, approximately $50 \%$ are attributed to male factors [2].

When considering male factors for infertility, the World Health Organization (WHO) has established standardized parameters, which outline the characteristics of healthy, normal spermatozoa. The WHO sets standards for sperm motility, morphology, and concentration [2]. Physicians and fertility laboratories thereby compare the semen characteristics of the patient against these standardized parameters to identify any deficiencies.

However, in the last decade there have been rising concerns about factors undermining male fertility potentials which may lie outside the realm of WHO standards. DNA damage in spermatozoa, and subsequent effects of such damage on male reproductive ability, is one of these factors which has been studied in the past few years [3]. Because half of the offspring's DNA is originating from paternal unit, it is of utmost importance to consider the detrimental effects of sperm chromatin and DNA damage on reproduction.

*Address correspondence to this author at the Center for Reproductive Medicine, Cleveland Clinic, Desk A19.1, 9500 Euclid Avenue, Cleveland, Ohio 44195, USA; Tel: (216) 444-9485; Fax: (216) 445-6049; E-mail: agarwaa@ccf.org
This chapter will open with a discussion of human spermatozoa DNA structure and organization. Next, DNA damage and the causative sources will be analyzed. Subsequently, the clinical significance of DNA damage and available testing options will be reviewed, followed finally, by a discussion of both inherent mechanisms of DNA repair and pharmaceutical methods used to reduce DNA damage.

\section{STRUCTURE OF HUMAN SPERM DNA}

Human spermatozoon is a highly organized unit which attains its structural and functional integrity through a very unique packaging system. The coiling of human sperm DNA material is mediated by specific proteins which provide control over condensation and decompression in a time dependent manner. The DNA must be decompressed to expose reading frames for protein synthesis at certain stages of embryo development, yet must be compressed to protect it from degradation and damage [4]. This balance is synchronized by the structural organization of the DNA.

Mammalian sperm DNA is structurally different in organization from that of somatic cell. The majority of sperm DNA is coiled into highly condensed toroids due to the incorporation of protamines, a smaller percent is bound to histones in a much looser form, and the remaining DNA is attached to the sperm nuclear matrix at matrix attachment regions (MARs) at intervals of roughly $50 \mathrm{~kb}$ throughout the genome [5].

\section{Protamine Bound DNA}

The most compressed organization of spermatozoa DNA exists in toroids. During maturation process, majority of the histone proteins associated with DNA are replaced with protamines. This protamine associated DNA allows for tighter condensation and makes the DNA resistant to nuclease digestion. The protamines contain large bands of 


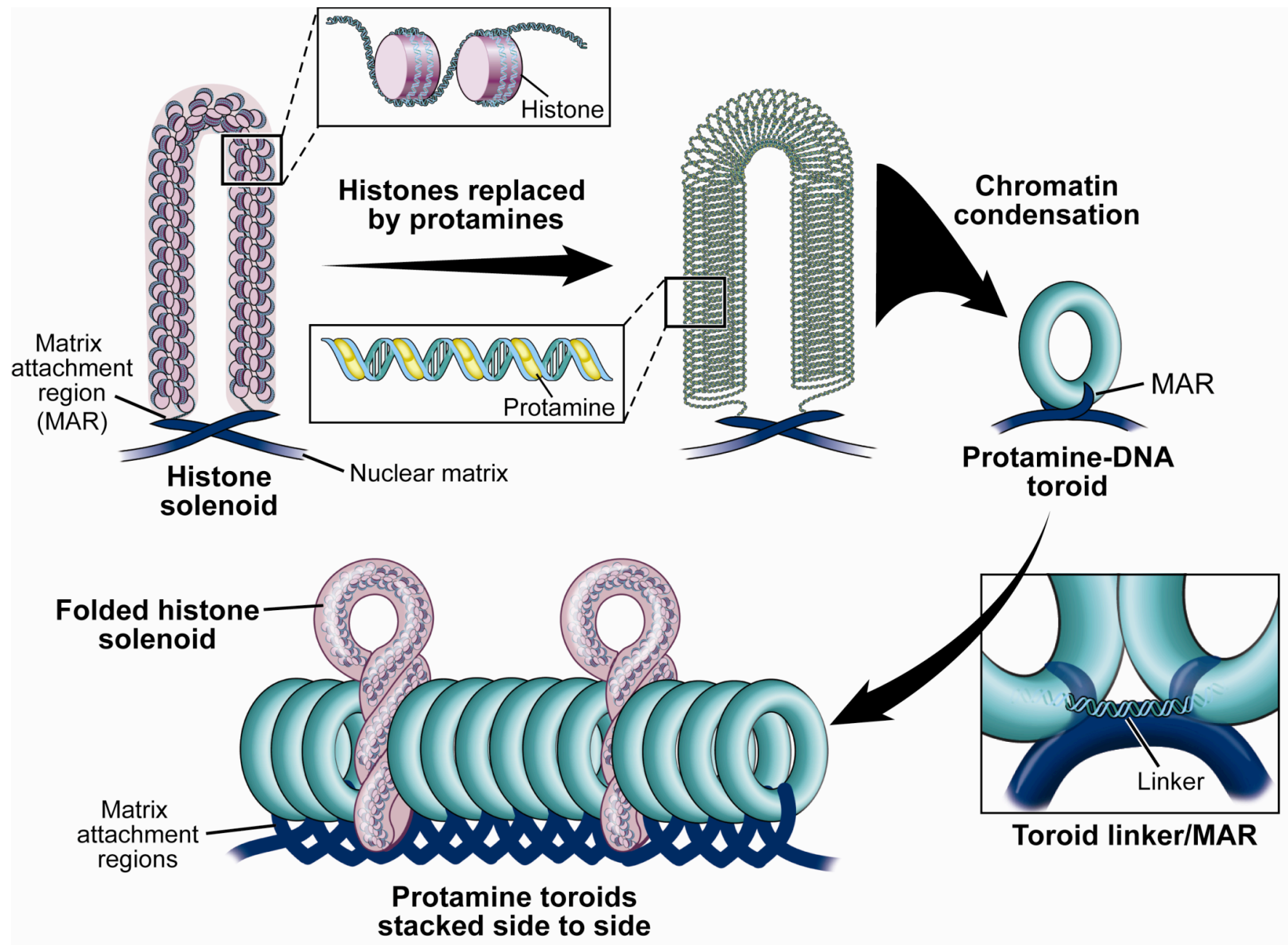

Fig. (1). Sperm DNA Organization. Spermatozoa DNA is organized into three main domains: the majority of the sperm DNA is coiled into DNAse-insensitive toroids that are stacked side to side to maximize compaction. This toroid structure is held stable due to the presence of protamines which neutralize the repulsion between the phosodiester backbones. A smaller amount of DNA is associated with histones present in the spermatozoa, with the remaining DNA attached to the nuclear matrix at Matrix Attachment Regions. Adapted from Ward (2010) [5].

positively charged arginine residues that neutralize the negative phosphodiester backbone of the DNA. Such interaction minimizes the repulsion within the DNA backbone, allowing it to double back and fold up onto itself and thus creating the highly compact and tightly wound toroids [5]. These toroids are lined up side by side to provide maximum surface area allocation (Fig. 1).

Protamines provide not only substantial compaction to DNA structure but also protection against damage. Mammalian protamines are rich in cysteine residues forming.

Intermolecular disulfide cross-links that render the sperm chromatin more resistant to mechanical disruption than that of somatic cells [6].

DNA compaction is considered a gene expression control mechanism. Specifically, this compaction blocks access to reading frame and thereby causes silencing of gene expression during spermatogenesis [7]. Once the spermatozoon fuses with the oocyte, the protamines are completely replaced with histone proteins provided by the oocyte within the first four hours. Such replacement allows for the paternal chromatin to have increased accessibility for protein generation [8]. This reconstruction process also indicates that the function of protamine toroids is to protect the sperm during its journey in male and female reproductive tract till the time of fertilization and they do not play a role in embryonic development. This has been demonstrated when round spermatids lacking protamine condensation were directly injected into mouse oocytes, resulting in development of normal fertile mice [9].

\section{Histone Associated DNA}

The second most prevalent form of sperm DNA structure is histone bound DNA. Approximately 4\% of the DNA in mature spermatozoa is bound to histones, although between $2-15 \%$ of the total sperm chromatin can be bound to histones in various mammalian species [10]. Histones are primarily associated with gene promoter site. Entire gene families, which are vital for spermiogenesis and early fertilization events, are preferentially associated with histones in human spermatozoa [10-12]. More specifically, human histones are found in association with miRNA clusters, $H O X$ gene 
clusters, and the promoters of stand-alone developmental transcription and signaling factors [10].

While DNA binding to histones allows for more accessible reading frame, such accessibility renders DNA more vulnerable to degradation by nuclease activity. Most sperm DNA accessible sites are at the linker regions between protamine toroids in each chromatin fiber, due to the fact that these regions are extremely nuclease sensitive and contain a large amount of histone-bound DNA [8].

Furthermore, histones in the sperm DNA are not replaced by those found in the oocyte post fertilization. This suggests that an inflicted damage to histone bound sperm DNA will be transmitted to the embryo without detection and possible modification. This may be particularly harmful due to the fact that most of the DNA bound to histones is significantly rich in gene clusters responsible for early development [8, $10,13]$.

\section{Matrix Attachment Regions}

The third and final form of spermatozoa DNA organization is the nuclear matrix attachment region (MAR). The MARs are segments of DNA attaching the loop domains of the chromatin to the proteinaceous nuclear matrix. These MARs are no larger than 1000 base pairs and located between each protamine toroid anchoring the toroids into place. Due to this role they are often called the toroid linkers (Fig. 1) [14]. These toroid linkers contain histone and are thereby extremely sensitive to nuclease activity. In addition to providing association between the DNA and the nuclear matrix, these MARs also function as a checkpoint for sperm DNA integrity after fertilization. These MARs, along with other histone bound DNA, are directly derived from the paternal genetic material and are inherent to the embryo and vital for proper development [5]. Thus, artificial reproductive techniques (ART) should focus on minimizing damage and maintain genetic integrity of these regions to ensure proper embryo maturation.

\section{TYPES OF DNA DAMAGE}

\section{Single and Double Strand Breaks}

Strand breaks are the main form of DNA damage incurred in spermatozoa. These breaks are in part due to the inherent nature of chromatin compaction and organization. The formation of the toroids induces a high degree of torsional stress on the phosphodiester backbone of DNA. This tension must be resolved by creation of single strand breaks periodically along the genetic material. These periodic breaks allow DNA to fold up onto itself and gain a higher extent of compaction.

However, whereby single strand breaks may be a regular component of the DNA maturation process, the associated formation of double strand breaks is not. These double strand breaks are not easily detected and fixed by the topoisomerase II variants found in spermatozoa [15]. Also, it should be noted that the presence of strand breaks increases with age of the male that is also inversely correlated with apoptosis rates in spermatozoa [16]. Thus with ageing, the number of spermatozoa with DNA strand breaks increases, along with insufficient mechanisms that are supposed to detect and eliminate such cells. Obviously, extra precautions should be taken into account when using spermatozoa from older males for ART.

\section{Alternation of Bases}

Base modifications are the second most prevalent form of spermatozoa DNA damage after strand breaks. These base alterations result in improper reading of the base sequences, and incorrect protein production. These changes are due to either additions to the base sequence or subtraction of the necessary bases. Both conditions lead to frame shifts and subsequently affect the entire protein produced.

Base supplementations, or adducts, are commonly thymidine glycol additions and fappy $\mathrm{G}$ incorporations. These additions can be repaired by base excision repair (BER) mechanisms, however it is often difficult for these mechanisms to detect the additions until further down the line, where the inappropriate base sequences produce incorrect proteins [17]. Because embryo development is a very time sensitive process, this incorrect protein may have a devastating impact on the proper formation and maturation of the embryo, and may lead to early termination and apoptosis.

Base deletion and related mechanisms also lead to improper protein synthesis. The most common forms of such mechanisms are formation of intrastrand bridges and pyrimidine dimerization. These two modifications cause the reading frames of the DNA to shorten and unnecessarily skip several base pairs in-between. Again, this can result in formation of improper protein residues and can have a lasting effect on the entire maturation process. There are however repair mechanisms in the spermatozoa and oocyte which can help reverse such cross linkages and expose the entire DNA strand. Tyrosyl-DNA phosphodiesterase 1 (TDP-1) can repair intrastrand bridges, while nucleotide excision repair (NER) mechanisms reverses pyrimidine dimerization [17, $18]$.

In addition to base adducts and intrastrand bridges, there are several chemical modifications which can have similar results. Oxidation of guanosine sites, abasic sites, and phosphorylation of bases can all lead to mismatched pairs, frame shits, and erroneous transmission of genetic data $[17,18]$.

\section{ORIGIN OF SPERM DNA DAMAGE}

\section{Internal Factors}

\section{A. Defects in Spermatozoa Maturation Process}

Before looking at external sources of DNA damage, the inherent nature of the spermatozoa and its maturation must be scrutinized. Generally, sperm have small amount of cytoplasm and hence limited reserve of cytoplasmic antioxidants. Such property leaves the spermatozoa particularly vulnerable to oxidative stress brought upon by free radical reactive oxygen species (ROS). Also the plasma membrane of the spermatozoa is rich in unsaturated fatty acids which maintain the fluidity of the membrane. However, such high fatty acid content attracts unwarranted ROS on-slaught. This mechanism perpetuates oxidative stress resulting in peroxidation of 
the plasma membrane, causing substantial damage to the sperma-tozoa and its defense mechanisms [19].

As aforementioned, sperm DNA packaging process is a unique and highly complicated mechanism. Such complexity exposes the DNA to damage by means of improper execution of any of the steps involved. For instance, irregular protamination can result in increased torsional stress leading to additional strand breaks which, as previously discussed, impacts DNA integrity, protein production, and embryo development [20].

\section{Oxidative Stress}

The second most common proponent of DNA damage is oxidative stress. Oxidative stress occurs when there is an excess production of reactive oxygen species (ROS), which exceeds the neutralizing capabilities of naturally present cellular antioxidants. Studies have shown that between 25 and $40 \%$ of infertile men have semen with elevated amounts of ROS resulting in a cascade event of lipid peroxidation and degeneration of cellular macromolecules [21]. As aforementioned, the general composition of the spermatozoa cellular membrane along with the limited antioxidant availability makes these cells specifically prone to OS.

Furthermore, the presence of leukocytospermia and varicocele are both correlated with elevated ROS levels in semen. Varicocele may affect seminal DNA damage levels via two mechanisms: directly through increased scrotal temperature or indirectly though increased production of ROS. Most recently, Smith et al. showed that the DNA fragmentation index (DFI) values were significantly higher in patients with varicocele, either with normal (DFI, 20.7 4.0) or with abnormal (DFI, 35.5, 9.0) semen profile, compared with controls (DFI, 7.1, 0.9). Furthermore, ROS levels were significantly higher $(\mathrm{P}<0.01)$ in both groups of patients with varicocele [22]. On the other hand, leukocytospermia contributes to an increased release of pro-inflammatory mediators (cytokines) which altering the regulatory mechanisms of spermiogenesis and subsequently attributing to DNA aberration [23].

OS DNA damage is marked by detection of 8-hydroxy-2deoxyguanosine (8-OHdG). $8-\mathrm{OHdG}$ is an adduct serving as a biological marker, attaching to the damaged DNA and representing an altered DNA structure which potentially could lead to additional DNA breaks [24]. Loft et al. showed that $8-\mathrm{OhdG}$ can be used as a reliable marker for DNA damage and also concluded that the levels of $8-\mathrm{OHdG}$ are inversely correlated to the likelihood of pregnancy occurring within a single menstrual cycle $(\mathrm{P}<0.01)[25]$.

\section{Abortive Apoptosis}

A third, and final, mechanism of internal DNA damage to spermatozoa is abortive apoptosis. Apoptosis is a programmed cellular death, a natural process required to remove old and senescent sperm. During spermiogenesis, apoptosis plays a key role in adjusting the appropriate number of proliferating germ cells [26]. Fas cell surface proteins help regulate the apoptosis in spermatozoa. These Fas surface proteins and their associated ligands can serve as markers for the rate of apoptosis.
In men with abnormal semen parameters, the percentage of Fas-positive spermatozoa may be as high as 50\%. This may be attributed in part to the inability of the Sertoli cells to trigger Fas-ligand production and carry out apoptosis [27, 28]. Therefore, these Fas positive cells that escape apoptosis, can successfully mature and their damaged DNA may be incorporated into the gene pool.

In addition to improper Fas-ligand production, there may be defects in the actual apoptosis pathway itself such as improper caspase activation. Caspases, also know as cysteine-aspartic proteases or cysteine-dependent aspartatedirected proteases, are a family of cysteine proteases which are involved in the initial steps of the apoptosis pathway. They begin a cascade of events which culminates into programmed cell death. However, Kim et al. found that if the pathway from $\mathrm{C} 8 / 9$ to $\mathrm{C} 3$ to caspase-activated deoxyribonuclease (CAD) is improperly carried out, apoptosis of spermatozoa is inhibited [29]. The above mechanisms may underlie inefficient or abortive apoptosis increasing the proportion of sperm with damaged DNA previously destined for death.

\section{External Factors of DNA Damage}

External factors have been also implicated as predisposing factors for sperm DNA damage. Lifestyle behaviors, radiation, heat exposure, medications and substance abuse are examples of such factors. Lifestyle choices such as cigarettes, medications, recreational drugs, and caffeine all have an impact on the amount of DNA damage found in spermatozoa. Many of these products contain chemicals which are either directly linked with increases in strand breaks or indirectly via secondary oxidative methods.

Cigarette smoking is an example of external factors that causes sperm DNA damage via oxidative stress. Many of the metabolites in cigarettes smoke trigger release of chemical inflammatory mediators such as interleukin- 6 and interleukin-8 [30]. These mediators can recruit additional leukocytes, resulting in increased ROS formation in the semen. Two additional cigarette metabolites, vinyl chloride and benzopyrene, can also lead to an increased attachment of DNA adducts [17]. These adducts contribute to mismatched pairs, improper DNA replication, and incorrect protein synthesis. A prospective study of 65 males visiting an infertility clinic found that infertile men who smoked had significantly higher percentage DNA fragmentation index than those who did not [31].

In addition to cigarettes, many medications have been shown to have negative effects on semen DNA parameters. Alkylating agents used in cancer chemotherapy are notoriously known for sperm DNA damaging sources, whose effects persist several months after cessation [32]. Drugs such as fludarabine, cyclophosphamide and buslphan have variable effects ranging from reduced testicular volume, lower testosterone concentrations, to oligozoospermia, and elevated follicle stimulating hormone and luteinizing hormone production [33].

Other drugs, such as cocaine and caffeine can also degrade spermatozoa DNA. Cocaine exposure increases sperm DNA strand breaks resulting in apoptosis [34]. Furthermore, 
caffeine consumption is associated with an increase in double strand breaks in sperm DNA [35]. Caffeine may also lead to an inactivation of H2AFX through the inhibition of kinases related to DNA repair including ATM, ATR, and DNA protein kinase $\mathrm{C}$ [35].

\section{IMPLICATIONS OF DAMAGED DNA IN SPERM RELATED TO ART APPLICATIONS}

Artificial reproductive technologies (ART) encompass a large variety of methods which can be used to facilitate the achievement of fertilization, implantation, and successfully complete pregnancy. The three most common methods are intrauterine insemination (IUI), in vitro fertilization (IVF), and intracytolasmic sperm injection (ICSI). Unfortunately, although there have been substantial developments in these techniques, damage to the spermatozoa DNA can still have a large impact on their outcomes. Several studies have shown a negative correlation between ART and DNA damage.

\section{Intrauterine Insemination (IUI)}

Studies have shown that there is poor pregnancy outcome in women inseminated with a semen sample with $>12 \%$ sperm with fragmented DNA and miscarriages in those with moderate degrees of DNA damage (10-12\%). In a study on 119 couples comprised a total of 154 IUI cycles (natural and stimulated) found that an increased rate of miscarriages and pregnancy failure correlated with the level of sperm DNA damage. This damage may have been brought upon by defective chromatin organization, ineffective apoptosis, and oxidative stress [36].

Furthermore, Bungum et al. also conducted a similar study, analyzing at 387 IUI cycles. Their results confirmed those found earlier, with a pregnancy rate of $19 \%$ when the DFI value was $<30 \%$, compared to a rate of only $1.5 \%$ when the DFI was $>30 \%$. These results indicate that the DFI value is a reliable and an accurate independent predictor of fertility outcomes [37].

\section{IVF/ICSI}

In addition to studies on IUI, there has been research conducted on the link between sperm DNA and IVF/ICSI. The increased levels of DNA damage are associated with a significantly higher rate of pregnancy loss after IVF or ICSI. A recent meta-analysis of 2549 cycles of IVF or ICSI involving 640 pregnancies and 122 failures, showed that sperm genetic damage was significantly associated with pregnancy loss $(\mathrm{P}<0.0001)$. It was also found that with abnormal sperm DNA damage, the miscarriage rate increases to $37 \%$ compared to an average rate of $18 \%$. In comparison this rate fell to $10 \%$ when the testing results are negative [38].

Moreover, Virro et al. concluded in a study on 249 couples undergoing IVF/ICSI that semen samples with a DFI value under $33 \%$ has a significantly greater chance of initiating a pregnancy, lower miscarriage rates, and an increase of ongoing pregnancies at week $12(47 \%$ vs. $28 \%)$ than those with a DFI value higher than 33\% [39]. These studies show the importance of DFI as a valuable measure and point to the importance of DNA integrity in pregnancy initiation and also in long-term viability of the embryo and success of the pregnancy.

\section{DNA DAMAGE TESTING AND STRATEGIES TO MINIMIZE DNA DAMAGE}

\section{DNA Damage Testing}

There have been many established testing protocols for analyzing DNA damage in spermatozoa. Each of these techniques can detect specific aspects of the damage varying from DNA adducts to strand breaks. However, most of the tests do not have standardized thresholds or lack good sensitivity and specificity. This section will quickly review some the assays available.

Terminal deoxynucleotidyl transferase dUTP nick end labeling (TUNEL) is an assay used for detecting stand breaks. It quantifies the incorporation of dUTP at single strand breaks and double strand breaks via template independent TdT.

Single cell gel electrophoresis assay (also known as COMET assay) is another test used to determine the amount of strand breaks. The cells are suspended in solution, lysed in an alkaline media, and then subjected to electrophoresis followed by DNA staining. This test lacks a standardized protocol or threshold and therefore results vary from laboratory to laboratory As with TUNEL, COMET also results in destruction of the tested cells thereby resulting in unusable sperm for ART.

In situ nick translations assay (NT) quantifies the incorporation of dUTP at single strand breaks via template dependent DNA polymerase I. However, there has been no proven correlation between this assay and fertilization during in vivo studies, and it lacks sensitivity/ specificity.

Sperm chromatin structure assay (SCSA) measures DNA denaturation following heat or acid treatment via flow cytometry fluorescence shifts. This assay measures the widely used concept "DNA fragmentation index", and is a well published and popular assay used today.

There are also various independent tests, each has a highly specific and unique target for testing. Such tests include ELISA (enzyme-linked immunosorbent assay), 3D and 2D FISH (florescent in situ hybridization) which is used to locate specific chromatin territories in sperm cells during interphase, and lastly, high-performance liquid chromatography. These tests are not used on a common basis and may be ordered for a specific patient's needs.

\section{Strategies to Minimize DNA Damage}

There are many inherent and external means and strategies to minimize the damage to sperm DNA. Sperm preparation techniques will be discussed first, followed by repair mechanism of DNA damage performed by oocyte.

There are various sperm preparation methods help reduce DNA damage during ART. The swim-up method and density-gradient centrifugation have been proven to have an increased post-IVF fertilization rate after use of these selected sperm [40, 41]. However, the swim-up method shows better results in terms of DNA integrity [42]. Also, glass 
wool filtration yields sperm with significantly decreased \% DFI integrity when compared with raw semen samples [43].

Furthermore, sperm preparation techniques can have an effect on sperm DNA. The intensity of centrifugation can lead to DNA damage as well as removal of the seminal plasma undermining the protective armor from the semen. Seminal plasma contains enzymatic and non-enzymatic ROS scavengers such as $\mathrm{Cu}, \mathrm{Zn}$, superoxide dismutase and catalase as well as ascorbate, urate, albumin, glutathione and taurine [44]. Removal of this protective plasma in addition to the heat generated during centrifugation can have a negative effect on sperm DNA integrity. Cryopreservation can also induce sperm DNA damage through the freezing and thawing protocol $[45,46]$. Spermatozoa which were preserved without cryoprotectants revealed more fragmented DNA [47]. Flash-freezing in liquid nitrogen without cryopreservative represents the most appropriate method for human sperm cryopreservation in terms of post-thaw DNA integrity [48].

There are of course other extraneous methods to reduce DNA damage, such as lifestyle modification, stopping smoking, avoiding hyperthermia, limiting caffeine intake, and beginning an antioxidant regimen to reduce damage.

In addition to repair mechanisms and lifestyle changes available in the male, there are some limited repair mechanisms innate to the oocyte. These can play a vital role in the proper development of the embryo post fertilization. There is DNA polymerases present in the oocyte which can correct some of the defects in the DNA. Between the period of sperm entry into the cytoplasm and the beginning of the next $\mathrm{S}$ phase, DNA damage can be repaired by the oocyte via pre and post-replication mechanisms. Various repair mechanisms available in the oocyte include non-homologous end joining, homologous recombination, mismatch repair, and nucleotide/base excision repair [17]. These mechanisms are used to rescue the genetic integrity of the paternal genome. However, maternal age must be kept in mind, as it is found that the increased maternal age is correlated with a decrease in the store of mRNA for many of these repair mechanisms, marking a decline in the efficiency of DNA repair [49].

\section{CONCLUSIONS}

Today's advances in artificial reproductive technologies allow individuals who were previously without hope to enjoy the gifts of parenthood and have an enriched family life. These techniques allow infertile couples to manipulate the means of reproduction and bypass barriers which would otherwise make them unable to fertilize, implant, and have a successful pregnancy term.

With over 50 percent of the infertility cases stemming from the paternal origin, it is of utmost importance to get a comprehensive picture of the defects in spermatozoa. Unfortunately, current World Health Organization protocol only outlines an analysis of sperm motility, viability, count, and morphology when studying spermatozoa from infertile men [2].

The impact of spermatozoa DNA damage can be substantial and long lasting. There are many sources of this damage, some internal and inherent, and some due to external lifestyle factors. The future of DNA damage analysis should revolve around standardizing the testing protocols such that the significance of DNA damage pertaining to the outcomes of ART can be explicitly defined. It is only after such standardization of testing, as well as generation of concrete data, that DNA analysis can be incorporated in the WHO guidelines.

\section{ACKNOWLEDGEMENTS}

None Declared.

\section{CONFLICT OF INTEREST}

None Declared.

\section{REFERENCES}

[1] Dunson DB, Baird DD, Colombo B. Increased infertility with age in me and women. Obstet Gynecol 2004; 103: 51-6.

[2] World Health Organization DoRHaR. WHO laboratory manual for the examination and processing of human semen. $5^{\text {th }}$ ed. 2010.

[3] Marchettini P, Solaro C. To become a pain specialist one has to understand the nervous system, yet the specialists of the nervous system still have a long way to go before understanding pain. Neurol Sci 2007; 28: 161-2.

[4] Fuentes-Mascorro G, Serrano H, Rosado A. Sperm chromatin. Arch Androl 2000; 45: 215-25.

[5] Ward WS. Function of sperm chromatin structural elements in fertilization and development. Mol Hum Reprod 2010; 16: 30-6.

[6] Sotolongo B, Lino E, Ward WS. Ability of hamster spermatozoa to digest their own DNA. Biol Reprod 2003; 69: 2029-35.

[7] Martins RP, Ostermeier GC, Krawetz SA. Nuclear matrix interactions at the human protamine domain: a working model of potentiation. J Biol Chem 2004; 279: 51862-8.

[8] Ajduk A, Yamauchi Y, Ward MA. Sperm chromatin remodeling after intracytoplasmic sperm injection differs from that of in vitro fertilization. Biol Reprod 2006; 75: 442-51.

[9] Ogura A, Matsuda J, Yanagimachi R. Birth of normal young after electrofusion of mouse oocytes with round spermatids. Proc Natl Acad Sci USA 1994; 91: 7460-2.

[10] Hammoud SS, Nix DA, Zhang H, Purwar J, Carrell DT, Cairns BR. Distinctive chromatin in human sperm packages genes for embryo development. Nature 2009; 460: 473-8.

[11] Ostermeier GC, Goodrich RJ, Diamond MP, Dix DJ, Krawetz SA. Toward using stable spermatozoal RNAs for prognostic assessment of male factor fertility. Fertil Steril 2005; 83: 1687-94.

[12] Arpanahi A, Brinkworth M, Iles D, et al. Endonuclease-sensitive regions of human spermatozoal chromatin are highly enriched in promoter and CTCF binding sequences. Genome Res 2009; 19 : $1338-49$.

[13] van der Heijden GW, Ramos L, Baart EB, et al. Sperm-derived histones contribute to zygotic chromatin in humans. BMC Dev Biol 2008; 8: 34.

[14] Shaman JA, Yamauchi Y, Ward WS. The sperm nuclear matrix is required for paternal DNA replication. J Cell Biochem 2007; 102: 680-8.

[15] Spano M, Seli E, Bizzaro D, Manicardi GC, Sakkas D. The significance of sperm nuclear DNA strand breaks on reproductive outcome. Curr Opin Obstet Gynecol 2005; 17: 255-60.

[16] Singh NP, Muller CH, Berger RE. Effects of age on DNA doublestrand breaks and apoptosis in human sperm. Fertil Steril 2003; 80: 1420-30.

[17] Menezo Y, Dale B, Cohen M. DNA damage and repair in human oocytes and embryos: a review. Zygote 2010; 4: 357-65.

[18] Erenpreiss J, Spano M, Erenpreisa J, Bungum M, Giwercman A Sperm chromatin structure and male fertility: biological and clinical aspects. Asian J Androl 2006; 8: 11-29.

[19] Twigg J, Fulton N, Gomez E, Irvine DS, Aitken RJ. Analysis of the impact of intracellular reactive oxygen species generation on the structural and functional integrity of human spermatozoa: lipid 
peroxidation, DNA fragmentation and effectiveness of antioxidants. Hum Reprod 1998; 13: 1429-36.

[20] Agarwal A, Said TM. Role of sperm chromatin abnormalities and DNA damage in male infertility. Hum Reprod Update 2003; 9: 33145.

[21] Padron OF, Brackett NL, Sharma RK, Lynne CM, Thomas AJ, Jr., Agarwal A. Seminal reactive oxygen species and sperm motility and morphology in men with spinal cord injury. Fertil Steril 1997; 67: $1115-20$.

[22] Smith R, Kaune H, Parodi D, Madariaga M, et al. Increased sperm DNA damage in patients with varicocele: relationship with seminal oxidative stress. Hum Reprod 2006; 21(4): 986-93.

[23] Kasturi SS, Osterberg C, Tannir J, Brannigan RE. The effect of genital tract infection and inflammation on male infertility. In: Lipshultz L, Howard S, Niederberger C, Eds. Infertility in the male, $4^{\text {th }}$ ed. UK: Cambridge University Press $2009 ; 295-300$.

[24] Aitken RJ, De Iuliis GN. On the possible origins of DNA damage in human spermatozoa. Mol Hum Reprod 2010; 16: 3-13.

[25] Loft S, Kold-Jensen T, Hjollund NH, et al. Oxidative DNA damage in human sperm influences time to pregnancy. Hum Reprod 2003; 18: 1265-72.

[26] Sinha Hikim AP, Swerdloff RS. Hormonal and genetic control of germ cell apoptosis in the testis. Rev Reprod 1999; 4: 38-47.

[27] Huszar G, Sbracia M, Vigue L, Miller DJ, Shur BD. Sperm plasma membrane remodeling during spermiogenetic maturation in men: relationship among plasma membrane beta 1,4-galactosyltransferase, cytoplasmic creatine phosphokinase, and creatine phosphokinase isoform ratios. Biol Reprod 1997; 56: 1020-4.

[28] Francavilla S, D'Abrizio P, Rucci N, et al. Fas and Fas ligand expression in fetal and adult human testis with normal or deranged spermatogenesis. J Clin Endocrinol Metab 2000; 85: 2692-700.

[29] Kim JM, Ghosh SR, Weil AC, Zirkin BR. Caspase-3 and caspaseactivated deoxyribonuclease are associated with testicular germ cell apoptosis resulting from reduced intratesticular testosterone. Endocrinology 2001; 142: 3809-16.

[30] Sepaniak S, Forges T, Gerard H, Foliguet B, Bene MC, MonnierBarbarino $\mathrm{P}$. The influence of cigarette smoking on human sperm quality and DNA fragmentation. Toxicology 2006; 223: 54-60.

[31] Saleh RA, Agarwal A, Sharma RK, Nelson DR, Thomas AJ. Effect of cigarette smoking on levels of seminal oxidative stress in infertile men: a prospective study. Fertil Steril 2002; 78: 491-9.

[32] Cai L, Hales BF, Robaire B. Induction of apoptosis in the germ cells of adult male rats after exposure to cyclophosphamide. Biol Reprod 1997; 56: 1490-7.

[33] Chatterjee R, Haines GA, Perera DM, Goldstone A, Morris ID. Testicular and sperm DNA damage after treatment with fludarabine for chronic lymphocytic leukaemia. Hum Reprod 2000; 15: 762-6.

[34] Li H, Jiang Y, Rajpurkar A, Dunbar JC, Dhabuwala CB. Cocaine induced apoptosis in rat testes. J Urol 1999; 162: 213-6.
[35] Eskenazi B, Wyrobek AJ, Sloter E, et al. The association of age and semen quality in healthy men. Hum Reprod 2003; 18: 447-54.

[36] Duran EH, Morshedi M, Taylor S, Oehninger S. Sperm DNA quality predicts intrauterine insemination outcome: a prospective cohort study. Hum Reprod 2002; 17: 3122-8.

[37] Bungum M, Humaidan P, Axmon A, et al. Sperm DNA integrity assessment in prediction of assisted reproduction technology outcome. Hum Reprod 2007; 22: 174-9.

[38] Zini A, Boman JM, Belzile E, Ciampi A. Sperm DNA damage is associated with an increased risk of pregnancy loss after IVF and ICSI: systematic review and meta-analysis. Hum Reprod 2008; 23: 2663-8.

[39] Virro MR, Larson-Cook KL, Evenson DP. Sperm chromatin structure assay (SCSA) parameters are related to fertilization, blastocyst development, and ongoing pregnancy in in vitro fertilization and intracytoplasmic sperm injection cycles. Fertil Steril 2004; 81: 1289-95

[40] Younglai EV, Holt D, Brown P, Jurisicova A, Casper RF. Sperm swim-up techniques and DNA fragmentation. Hum Reprod 2001; 16: 1950-3.

[41] Agarwal A, Allamaneni SS. Sperm DNA damage assessment: a test whose time has come. Fertil Steril 2005; 84: 850-3.

[42] Zini A, Finelli A, Phang D, Jarvi K. Influence of semen processing technique on human sperm DNA integrity. Urology 2000; 56: 1081-

[43] Larson KL, Brannian JD, Timm BK, Jost LK, Evenson DP. Density gradient centrifugation and glass wool filtration of semen remove spermatozoa with damaged chromatin structure. Hum Reprod 1999; 14: 2015-9.

[44] Siciliano L, Tarantino P, Longobardi F, Rago V, De Stefano C, Carpino A. Impaired seminal antioxidant capacity in human semen with hyperviscosity or oligoasthenozoospermia. J Androl 2001; 22 : 798-803.

[45] Donnelly ET, McClure N, Lewis SE. Cryopreservation of human semen and prepared sperm: effects on motility parameters and DNA integrity. Fertil Steril 2001; 76: 892-900.

[46] Labbe C, Martoriati A, Devaux A, Maisse G. Effect of sperm cryopreservation on sperm DNA stability and progeny development in rainbow trout. Mol Reprod Dev 2001; 60: 397-404.

[47] Linfor JJ, Meyers SA. Detection of DNA damage in response to cooling injury in equine spermatozoa using single-cell gel electrophoresis. J Androl 2002; 23: 107-13.

[48] Duty SM, Singh NP, Ryan L, et al. Reliability of the comet assay in cryopreserved human sperm. Hum Reprod 2002; 17: 1274-80.

[49] Hamatani T, Falco G, Carter MG, et al. Age-associated alteration of gene expression patterns in mouse oocytes. Hum Mol Genet 2004; 13: 2263-78.

This is an open access article licensed under the terms of the Creative Commons Attribution Non-Commercial License (http://creativecommons.org/licenses/by$\mathrm{nc} / 3.0 /$ ), which permits unrestricted, non-commercial use, distribution and reproduction in any medium, provided the work is properly cited. 